\title{
Melanonychia in HIV positive patients using zidovudine
}

\section{Melanoníquia em pacientes HIV positivos utilizando zidovudina}

\author{
Luana Lenzi, Astrid Wiens \& Roberto Pontarolo \\ Universidade Federal do Paraná \\ Email: pontarolo@ufpr.br
}

\begin{abstract}
The development of melanonychia is one of the adverse effects of the antiretroviral drug zidovudine. We evaluated 19 adult patients treated with zidovudine and showing nail lines, an unusual side effect sometimes reported. Liver, kidney and pancreas function, as the metabolic function of these patients were also evaluated. Immunological markers and the treatment's adherence rate were also obtained.

Our results suggest that melanonychia, as macrocytosis, may be associated with the use of antiretroviral therapy.
\end{abstract}

Keywords: zidovudine; HIV positive; melanonychia; macrocytosis.

\section{Resumo}

Um dos eventos adversos da zidovudina, um fármaco anti-retroviral, é o desenvolvimento de melanoníquia. Foram avaliados 19 pacientes adultos tratados com zidovudina evidenciando linhas ungueais, um efeito colateral incomum por vezes relatado. As funções hepática, renal, pancreática, bem como a função metabólica destes doentes também foram avaliados. Foram também obtidos marcadores imunológicos e a taxa de adesão ao tratamento.

Os nossos resultados sugerem que a melanoníquia, como a macrocitose, podem estar associadas à utilização de terapia anti-retroviral

Palavras-chave: zidovudina; HIV positivo; melanoníquia; macrocitose 


\section{Introduction}

Melanonychia is a brown to black pigmentation that arises in the nail plate due to the presence of melanin. It is more common in the black population, and usually appears in a longitudinal form (longitudinal melanonychia) ${ }^{[1]}$. Although rare, the pigmentation may involve the entire nail plate (total melanonychia), or adopt a transversal format (transverse melanonychia) ${ }^{[1,2]}$. The causes for melanonychia vary. It may result from inflammatory, traumatic (mycotic) origins, or from systemic diseases, medication, or from (benign) melanocyte hyperplasia ${ }^{[1]}$.

Among those drugs that may induce melanonychia are chemotherapeutic agents, antimalarials (chloroquine), antibiotics (minocycline), antiretroviral (lamivudine, zidovudine) and some metals (mercury, arsenic and gold salts). Pigmentation usually disappears following cessation of the therapy, but it might take a few years ${ }^{[1-3]}$. The present study reports cases from 19 patients using zidovudine who developed melanonychia in São José dos Pinhais - Paraná.

\section{Case Report}

From September 2011 to August 2012 nail longitudinal lines from brown in color to black were observed in 19 patients aged between 33 and 69 years (mean $=51.4, \pm$ 11.9), 8 were female and 11 male. Regarding ethnicity, 9 were white, 8 were black and 2 were of mixed-race. In relation to body weight, $52.6 \%$ weighed less than $60 \mathrm{Kg}$. All patients were adequately informed about the objectives of the study which was conducted according to the principles outlined in the Declaration of Helsinki and respective amendments.

All patients were HIV positive, under treatment with antiretrovirals, including zidovudine. The duration of use of zidovudine ranged from 0.5 to 6 years (average: $526.41 ; \pm 254.334$ days).

There was no other coincident pathology in these patients. 17 patients were tested for liver, pancreatic and kidney function as well as other serum dosages, in order to evaluate the metabolic functionality of these individuals. These all had normal values of glucose, amylase, total protein and albumin levels. Results from other determinations are shown in Table 1. The most significant changes were observed in the red blood cells which showed increased values of mean corpuscular volume $(\mathrm{MCV})(110.8 \pm 6.33, \min =101, \mathrm{Max}=122)$ and mean corpuscular hemoglobin $(\mathrm{MCH})(37.8 \pm 2.26$, $\min =35.6, \operatorname{Max}=43$ ) which indicates macrocytosis. Regarding immunological markers and prognosis of

\section{Introdução}

A melanoníquia é uma pigmentação de cor castanha a preta que surge na placa ungueal revelando a presença de melanina. É mais comum na população Africana, e geralmente aparece de forma longitudinal (melanoníquia longitudinal) ${ }^{[1]}$. Mais raramente, a pigmentação pode envolver a unha inteira (melanoníquia total), ou adotar um formato transversal (transversal melanoníquia) ${ }^{[1,2]}$.

As causas para a melanoníquia variam. Pode resultar de origens inflamatórias, traumáticas (micótico), ou de doenças sistémicas, reacções a medicamentos, ou da hiperplasia(benigna) melanocitária ${ }^{[1]}$.

Entre as drogas que podem induzir melanoníquia encontram-se os agentes quimioterapêuticos, antimaláricos (cloroquina), antibióticos (minociclina), anti-retrovirais (lamivudina, zidovudina) e alguns metais (mercúrio, arsénio e sais de ouro). A pigmentação desaparece geralmente após a cessação da terapia, mas isso pode demorar alguns anos ${ }^{[1-3]}$.

O presente estudo relata os casos de 19 pacientes submetidos a terapêutica com zidovudina, em São José dos Pinhais - Paraná, que desenvolveram melanoníquia.

\section{Relato}

Foram observadas, entre setembro de 2011 e agosto 2012 linhas longitudinais de cor castanha a preto nas unhas de 19 pacientes com idade entre 33 e 69 anos (média $=51,4, \pm 11,9$ ), sendo 8 do sexo feminino e 11 do sexo masculino. Quanto à etnia, nove eram brancos, 8 pretos e 2 mestiços. Em relação ao peso 52,6\% tinha menos de $60 \mathrm{~kg}$. Todos os pacientes foram devidamente informados sobre os objetivos do estudo, conduzido de acordo com os princípios enunciados na Declaração de Helsinki e respectivas alterações.

Todos os pacientes eram HIV positivos, em tratamento com anti-retrovirais, incluindo a zidovudina. O tempo de utilização de zidovudina, variou de 0,5 meses a 6 anos (média: 526,$41 ; \pm 254,334$ dias ).

Não existia, nestes pacientes, nenhuma outra patologia concomitante. Em 17 destes pacientes foram realizadas provas de função hepática, pancreática e renal e, determinados outros indicadores séricos para avaliação da sua funcionalidade metabólica. Todos apresentaram valores normais de proteína, glicose, amilase total e albumina. Os resultados de outras determinações são apresentados na Tabela 1. As alterações mais significativas foram observadas em células vermelhas do sangue, com valores aumentados de volume corpuscular médio $(\mathrm{VCM})(110,8 \pm 6,33, \min =101, \mathrm{Max}=122)$ e da hemoglobina corpuscular média (HCM) $(37,8 \pm 2,26$, $\min =35,6, \operatorname{Max}=43$ ) indicando macrocitose. 
infection, T lymphocyte CD4 + counts showed a minimum of 17 cells $/ \mathrm{mm} 3$ and a maximum of 1,236 cells/mm3 (mean 443.23; \pm 183.440 ). Viral load ranged from undetectable (less than 50 copies $/ \mathrm{mm} 3$ ) to 593,000 (average $47,202 \pm 65,870$ ). The rates of treatment adherence ranged from $48 \%$ to $100 \%$ (mean $=$ $88.3 \%, \pm 12.8)$.
Quanto aos marcadores imunológicos e ao prognóstico da infecção, o CD4 + contagem de linfócitos T mostrou um mínimo de 17 células $/ \mathrm{mm} 3$ e um máximo de 1236 células/mm3 (média de 443,23; $\pm 183,440$ ) e a carga viral variou de indetectável (menos de 50 copies $/ \mathrm{mm} 3$ ) para 593000 (média 47,202 $\pm 65,870$ ). A taxa de adesão ao tratamento variou de $48 \%$ a $100 \%$ (média $=88,3 \%, \pm 12,8$ ).

Table 1 - Results of laboratory tests of included patients.

Table 2 - Resultados laboratoriais dos pacientes incluidos no estudo

\begin{tabular}{|c|c|c|c|c|c|}
\hline & $\begin{array}{l}\text { REFERENCE VALUE } \\
\text { Valor de Referência }\end{array}$ & $\begin{array}{l}\text { LOW } \\
\text { Baixo }\end{array}$ & $\begin{array}{c}\text { NORM } \\
\text { AL }\end{array}$ & $\begin{array}{c}\text { HIGH } \\
\text { Elevado }\end{array}$ & $\begin{array}{l}\text { \% ALTERED } \\
\% \text { Alteração }\end{array}$ \\
\hline $\begin{array}{l}\text { CREATININE } \\
\text { CREATININA }\end{array}$ & $0.6-1.3 \mathrm{mg} / \mathrm{dL}$ & 1 & 16 & 0 & 5.9 \\
\hline ALT - Alanine aminotranferase & Até $55 \mathrm{U} / \mathrm{L}$ & 0 & 16 & 1 & 5.9 \\
\hline AST - aspartate aminotransferase & $5-34 \mathrm{U} / \mathrm{L}$ & 0 & 15 & 2 & 11.8 \\
\hline $\begin{array}{l}\text { Gama GT - gama- } \\
\text { glutamiltransferase }\end{array}$ & $<38 \mathrm{U} / \mathrm{L}$ & 0 & 16 & 1 & 5.9 \\
\hline $\begin{array}{l}\text { Alcalin phosphatase } \\
\text { Fosfatase alcalina }\end{array}$ & $53-141 \mathrm{U} / \mathrm{L}$ & 0 & 16 & 1 & 5.9 \\
\hline $\begin{array}{l}\text { LDH Lactic Dehydrogenase } \\
\text { desidrogenase lática }\end{array}$ & $125-243 \mathrm{U} / \mathrm{L}$ & 0 & 16 & 1 & 5.9 \\
\hline $\begin{array}{l}\text { Globulin } \\
\text { Globulina }\end{array}$ & $2.9-3.5 \mathrm{~g} / \mathrm{dL}$ & 1 & 14 & 2 & 17.6 \\
\hline $\begin{array}{l}\text { Total Bilirrubin } \\
\text { Bilirrubina total }\end{array}$ & $<1.2 \mathrm{mg} / \mathrm{dL}$ & 0 & 14 & 3 & 17.6 \\
\hline $\begin{array}{l}\text { Direct Bilirrubin } \\
\text { Bilirrubina direta }\end{array}$ & $<0.4 \mathrm{mg} / \mathrm{dL}$ & 0 & 15 & 2 & 11.8 \\
\hline $\begin{array}{l}\text { Indirect Bilirrubin } \\
\text { Bilirrubina indireta }\end{array}$ & $<0.8 \mathrm{mg} / \mathrm{dL}$ & 0 & 14 & 3 & 17.6 \\
\hline $\begin{array}{l}\text { Total Cholesterol } \\
\text { Colesterol Total }\end{array}$ & $<200 \mathrm{mg} / \mathrm{dL}$ & 0 & 12 & 5 & 29.4 \\
\hline $\begin{array}{l}\text { Triglycerides } \\
\text { Triglicerídeos }\end{array}$ & $<150 \mathrm{mg} / \mathrm{dL}$ & 0 & 12 & 5 & 29.4 \\
\hline $\begin{array}{l}\text { HDL cholesterol } \\
\text { Colesterol HDL }\end{array}$ & $>40 \mathrm{mg} / \mathrm{dL}$ & 1 & 16 & 0 & 5.9 \\
\hline $\begin{array}{l}\text { LDL cholesterol } \\
\text { Colesterol LDL }\end{array}$ & $\leqslant 129 \mathrm{mg} / \mathrm{dL}$ & 0 & 14 & 3 & 17.6 \\
\hline $\begin{array}{l}\text { Hematocrit }\left(0^{n} / \text { / }\right) \\
\text { Hematócrito }\left(\sigma^{n} / \text { / }\right)\end{array}$ & $40-52 \% / 37-46.9 \%$ & 5 & 12 & 0 & 29.4 \\
\hline 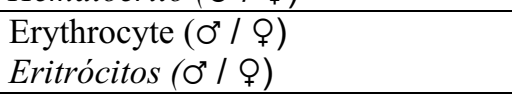 & $\begin{array}{c}4.35-5.87 / 3.9-5.23 \\
\text { million } / \mathrm{mm} 3\end{array}$ & 10 & 7 & 0 & 58.8 \\
\hline 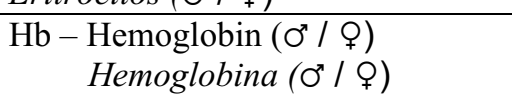 & $13.3-17.7 / 12.0-15.7 \mathrm{~g} / \mathrm{dL}$ & 4 & 13 & 0 & 23.5 \\
\hline $\begin{array}{c}\text { MCV - Mean Corpuscular Volume } \\
\text { Volume Corpuscular Médio }\end{array}$ & $80.0-97.0 \mathrm{fL}$ & 0 & 6 & 11 & 64.7 \\
\hline $\begin{array}{l}\text { MCH - Mean Corpuscular } \\
\text { Hemoglobin } \\
\quad \text { Hemoglobina corpuscular } \\
\text { média }\end{array}$ & $26,0-32.0 \mathrm{pg}$ & 0 & 5 & 12 & 70.6 \\
\hline $\begin{array}{r}\mathrm{CMCH}-\text { Concentration of } \mathrm{MCH} \\
\text { Concentração de } \mathrm{MCH}\end{array}$ & $31.0-36.0 \mathrm{~g} / \mathrm{dl}$ & 0 & 17 & 0 & 0 \\
\hline
\end{tabular}




\section{Discussion}

Zidovudine is a nucleoside analogue reversetranscriptase inhibitor. It was the first drug approved for the treatment of AIDS ${ }^{[4]}$, and has been used for years as monotherapy or dual therapy. It is currently part of triple regimens for the initiation of antiretroviral therapy (ART). It is one of the most frequently used antiretrovirals in the composition of therapeutic regimens for the treatment of HIV infection. Among the adverse effects are hepatic steatosis, lactic acidosis, and melanonychia may develop in some cases ${ }^{[5]}$. Previous studies have reported the presence of zidovudine in patients with melanonychia ${ }^{[6-8]}$. However, although widely used, only some patients develop melanonychia, and the etiology is still unknown. Laboratory tests in these patients indicated no perceptible change to justify this common manifestation of melanonychia.

Zidovudine is still associated with myelotoxicity, especially anemia, pancytopenia and macrocytosis. Macrocytosis is a result of both anemias due to deficiency of vitamin B12 or folic acid, common in cases of liver cirrhosis, alcohol abuse, hypothyroidism or as side effects of some drugs ${ }^{[9]}$. Macrocytosis indicated by increased values of MCV and consequently $\mathrm{MCH}$, occur due to the action of the drug on erythropoiesis by decreasing the cell division cycle time, yielding expanded cells [10]. In the past, most patients developed anemia when zidovudine monotherapy was the standard treatment.. Today, with increased potency and decreased toxicity achieved with the use of HAART (highly active antiretroviral therapy), anemia has ceased to be a problem for all patients, but macrocytosis still occurrs. A previous study associated macrocytosis, related to the chronic usage of zidovudine, with the treatment's compliance ${ }^{[10]}$. However, patients in this study had irregular compliances.

HIV infection can present several hematological abnormalities. These arise from the suppressive effect of HIV itself, ineffective hematopoiesis, infiltrative diseases of the bone marrow, peripheral consumption secondary to splenomegaly or immune dysregulation and nutritional deficiencies ${ }^{[11]}$. The chronic and the late stage of HIV infection, when accompanied by inadequate nutrition and poor absorption of micronutrients, may lead to protein-calorie deficiency and metabolic disorders. This highlights the deficiency of folic acid and vitamin B12. Thus, both macrocytosis, like melanonychia may be associated with multifactorial causes related to HIV infection and the use of ART ${ }^{[12]}$.

\section{Discussão}

A zidovudina é um nucleosídeo análogo inibidor da transcriptase reversa. Foi a primeira droga aprovada para o tratamento da SIDA ${ }^{[4]}$, e tem sido utilizado durante anos em monoterapia ou em terapia dupla. Hoje é parte de regimes triplos para o início da terapia antiretroviral (TARV). E um dos anti-retrovirais mais utilizados na composição dos regimes terapêuticos para o tratamento da infecção por HIV. Entre os efeitos adversos mais frequentes encontramos a esteatose hepática e a acidose láctica, mas a melanoníquia pode aparecer em alguns casos ${ }^{[5]}$. Estudos prévios têm relatado a presença de zidovudina em pacientes com melanoníquia ${ }^{[6-8]}$. Mas, apesar de amplamente utilizado, apenas alguns pacientes desenvolvem melanoníquia, e sua etiologia ainda é desconhecida e, os testes laboratoriais não permitiram encontrar justificação para esta manifestação. A zidovudina ainda está associada a mielotoxicidade, em especial à anemia, à pancitopenia $\mathrm{e}$ à macrocitose. A macrocitose é um resultado das anemias por deficiência de vitamina B12 e ácido fólico, comum em casos de cirrose hepática, alcoolismo, hipotiroidismo, ou relacionada com os efeitos secundários de alguns fármacos ${ }^{[9]}$. A macrocitose revelada por valores elevados de $\mathrm{MCV}$ e $\mathrm{MCH}$, pode ocorrer por acção da droga sobre a eritropoiese, a qual diminui o ciclo de divisão celular, promovendo a expansão das células ${ }^{[10]}$. A maioria dos pacientes desenvolvia anemia quando o tratamento com zidovudina em monoterapia era o padrão,. Hoje em dia, conseguidos o aumento da potència a par da diminuição da toxicidade através da utilização de HAART (terapia anti-retroviral altamente activa),a anemia deixou de ser um problema para os pacientes, mas a macrocitose ainda ocorre. Um estudo anterior associou a macrocitose relacionada com o uso crônico de zidovudina, à adesão terapêutica mas os pacientes utilizados neste estudo apresentavam compliances irregulares ${ }^{[10]}$.

A infecção pelo HIV pode envolver várias anormalias hematológicas que resultam do efeito supressor do próprio HIV, de uma hematopoiese ineficaz, de doenças infiltrativas da medula óssea, do consumo periféric secundário à esplenomegalia, ou da desregulação imunitária e deficiências nutricionais ${ }^{[1]}$. A infecção crônica e o estadio final dda infecção a HIV, quando acompanhada por nutrição inadequada e fraca absorção de micronutrientes, pode levar a deficiência protéicocalórica e a distúrbios metabólicos, o que sublinha a deficiência em ácido fólico e vitamina B12. Assim sendo, tanto a macrocitose como a melanoníquia parecem estar associadas com causas multifactoriais relacionadas com a infecção por HIV e a utilização de ART $^{[12]}$. 


\section{Conflict of Interest}

The authors declare that there is no financial or personal relationship that can be understood as representing a potential conflict of interest.

\section{Conflito de Interesses}

Os autores declaram a inexistência de qualquer tipo de relação financeira ou pessoal que possa representar um potencial conflito de interesses.

\section{References / Referências}

[1].Tosti A, Piraccini BM, De Farias DC. Dealing with melanonychia. Semin Cutan Med Surg 2009; 28(1):49-54.

[2].Pellicane BL, Rashid RM. Transverse melanonychia after radiation therapy. Cutis 2010; 85(5):239-41.

[3].Piraccini BM, Tosti A. Drug-induced nail disorders: incidence, management and prognosis. Drug Saf 1999; 21(3):187-201.

[4].Arts EJ, Quinones-Mateu ME, Albright JL. Mechanisms of clinical resistance by HIV-I variants to zidovudine and the paradox of reverse transcriptase sensitivity. Drug Resist Updat 1998; 1(1):21-8.

[5].Belda A, Borras-Blasco J, Arago M, Serra B.

[Zidovudine-induced melanonychia]. Farm Hosp
$2007 \cdot 31(2): 128-9$

[6].Depaoli M, Bedello P, Tamponi M Pigmentatione ungueale indotta da azidotimidina. Giorn Ital Dermatol 1989; 12471-72.

[7].Grau-Massanes M, Millan F, Febrer MI, Pujol C, Alegre VA, Salavert M, Navarro V, Aliaga A. Pigmented nail bands and mucocutaneous pigmentation in HIV-positive patients treated with zidovudine. JAm Acad Dermatol 1990; 22(4):687-8. [8].Sahai J, Conway B, Cameron D, Garber G. Zidovudine-associated hypertrichosis and nail pigmentation in an HIV-infected patient. Aids 1991; 5(11):1395-6.

[9]. Geené D, Sudre P, Anwar D, Goehring C, Saaïdia A, Hirschel B. Causes of macrocytosis in HIVinfected Patients not Treated with Zidovudine. J
Infect 2000;40:160-163.

[10]. Romanelli F, Empey K, Pomeroy C. Macrocytosis as an indicator of medication zidovudine adherence in patientes with HIV infection. AIDS Patient Care STDS 2002;16:405411.

[11]. Semba RD, Gray GE. Pathogenesis of anemia during human immunodeficiency virus infection. J Investig Med 2001; 49:225-239.

[12]. Oliveira OCA, Oliveira RA, Souza LR. Impacto do tratamento antirretroviral na ocorrência de macrocitose em pacientes com HIV/AIDS do município de Maringá, Estado do Paraná. Rev Soc Bras Med Trop 2011; 44(1):35-39. 